\title{
Equilibration of the Quark-Gluon Plasma at Finite Net-Baryon Density in QCD Kinetic Theory
}

\author{
Xiaojian Du® and Sören Schlichting $\odot$ \\ Fakultät für Physik, Universität Bielefeld, D-33615 Bielefeld, Germany
}

(Received 28 January 2021; revised 3 May 2021; accepted 25 June 2021; published 14 September 2021)

\begin{abstract}
We explore the out-of-equilibrium dynamics of the quark-gluon plasma at zero and finite net-baryon density based on an effective kinetic theory of quantum chromodynamics (QCD). By investigating the isotropization of the longitudinal pressure, we determine the relevant time and temperature scales for the onset of viscous hydrodynamics and quantify the dependence on the chemical composition of the quarkgluon plasma. By extrapolating our results to realistic coupling strength, we discuss phenomenological consequences regarding the role of the preequilibrium phase at different collision energies.
\end{abstract}

DOI: 10.1103/PhysRevLett.127.122301

Introduction.-High-energy heavy-ion collisions at the Relativistic Heavy-Ion Collider (RHIC) and the Large Hadron Collider (LHC) provide a unique opportunity to explore the properties of strong interaction matter under extreme conditions. During the first $\mathrm{fm} / c$ of the space-time evolution of heavy-ion collisions, the nonequilibrium plasma of quarks and gluons created in the collision of heavy nuclei undergoes an out-of-equilibrium evolution $[1,2]$, before relativistic viscous hydrodynamics becomes applicable to describe the ensuing collective expansion of the near-equilibrium quark-gluon plasma (QGP) [3,4]. During the collective expansion, the hot and dense QGP is described in terms of macroscopic properties such as temperature $T$, chemical potentials $\mu$, and fluid velocity $u^{\mu}$ and cools down until, on timescales $\sim 10 \mathrm{fm} / c$, the temperatures and densities approach the deconfinement transition of QCD, eventually leading to the production of final state hadrons measured in the RHIC and LHC experiments.

Even though the early preequilibrium phase represents a rather short period of time during the complex space-time evolution of a heavy-ion collision, it is imperative to understand the preequilibrium evolution of the QGP in order to establish a complete theoretical description of the reaction dynamics. To this end, significant progress has been made in recent years toward understanding the equilibration and onset of hydrodynamic behavior in high-temperature QCD plasmas, from holographic studies of supersymmetric theories [5-8] to weakly coupled YangMills [9-12] and QCD plasmas [13] as well as in various models [14-21].

Published by the American Physical Society under the terms of the Creative Commons Attribution 4.0 International license. Further distribution of this work must maintain attribution to the author(s) and the published article's title, journal citation, and DOI. Funded by SCOAP ${ }^{3}$.
Despite clear differences at the microscopic level, a common finding of different theoretical approaches is that macroscopic properties of the system, such as the nonequilibrium evolution of the energy-momentum tensor $T^{\mu \nu}$, can be accurately described by relativistic viscous hydrodynamics on a timescale $\tau_{\text {hydro }}$, long before the system approaches local thermal equilibrium on timescales $\tau_{\text {eq }} \gg \tau_{\text {hydro }}$. Strikingly, it has been also observed that, already at (very) early times $\tau \ll \tau_{\text {hydro }}$, the nonequilibrium evolution of macroscopic quantities may become insensitive to the details of the initial conditions and can be effectively described in terms of nonequilibrium attractors $[22,23]$, which provides an accumulation point for the evolution of macroscopic quantities in out-of-equilibrium plasmas $[24,25]$. While the existence of such attractors has been firmly established for different microscopic system undergoing a one-dimensional Bjorken expansion $[12,18,22,23,25-28]$, the study of nonequilibrium attractors remains an active research topic and has led to a number of interesting results $[21,26,29,30]$ aiming to understand and extend the range of applicability of effective macroscopic descriptions, such as relativistic viscous hydrodynamics.

Beyond theoretical interest, the memory loss of macroscopic quantities also plays an important role in the phenomenological description of the preequilibrium stage of high-energy heavy-ion collisions, e.g., to quantify the (approximate) amount of entropy production during the early preequilibrium stage which is directly connected to experimental measurements of the charged particle multiplicity in the final state [28] or to describe the space-time evolution of the preequilibrium plasma macroscopically in KoMPoST [10,31-33].

So far, investigations of the preequilibrium dynamics of the QGP have focused primarily on kinetic equilibration of pure glue QCD [9] with a recent extension to QCD [13] for charge neutral plasmas. By performing numerical 
simulations of the leading-order effective kinetic theory of QCD [34] for gluons and light flavor $(u, d, s)$ quarks, we extend the theoretical treatment of the preequilibrium description QGP for the first time to finite net-charge and net-baryon density. We investigate the existence of nonequilibrium attractors and describe their phenomenological consequences in the theoretical description of the preequilibrium stage. Within this Letter, we focus on the aspects most relevant to heavy-ion phenomenology and refer to our companion paper [35] for a detailed exposition of the theoretical framework and additional discussions.

Effective kinetic description of preequilibrium dynamics.-During the collision of heavy nuclei, a fraction of the energy and valence charge is deposited in a primordial plasma, providing the initial conditions for the subsequent preequilibrium evolution of the QGP. In high-energy collisions, the color glass condensate effective field theory of high-energy QCD [36,37] provides a theoretical description of the gluon-dominated initial state created at very early times [38-42]. Despite recent efforts to generalize this framework to include quark production [43-45], baryon stopping [46], and other corrections to the high-energy limit [47-49], so far, the chemical composition of the nonequilibrium plasma, as well as the structure of a nonequilibrium initial state in heavy-ion collisions at lower beam energies or the forward rapidity regions of high-energy collisions, is currently not well understood. We will therefore consider a rather generic parametrization of the initial phase-space distributions of gluons, quarks, and antiquarks [50]:

$$
\begin{gathered}
f_{g}\left(\tau_{0}, p_{T}, p_{\|}\right)=f_{g}^{0} \frac{Q_{0}}{\sqrt{p_{T}^{2}+\xi_{0}^{2} p_{\|}^{2}}} e^{-\left[2\left(p_{T}^{2}+\xi_{0}^{2} p_{\|}^{2}\right)\right] / 3 Q_{0}^{2}}, \\
f_{q_{f} / \bar{q}_{f}}\left(\tau_{0}, p_{T}, p_{\|}\right)=f_{q_{f} / \bar{q}_{f}}^{0} \frac{\sqrt{p_{T}^{2}+p_{\|}^{2}}}{\sqrt{p_{T}^{2}+\xi_{0}^{2} p_{\|}^{2}}} e^{-\left[2\left(p_{T}^{2}+\xi_{0}^{2} p_{\|}^{2}\right)\right] / 3 Q_{0}^{2}},
\end{gathered}
$$

where the initial abundance of the $N_{f}=3$ light flavor quarks and antiquarks is determined by the sum of the contributions from valence quark stopping $\left(f_{v}^{0}\right)$ and quark or antiquark pair production $\left(f_{0}^{s}\right)$ as

$$
\begin{array}{ll}
f_{u}^{0}=\frac{2 n_{p}+n_{n}}{3\left(n_{p}+n_{n}\right)} f_{v}^{0}+\frac{1}{2 N_{f}} f_{s}^{0}, & f_{\bar{u}}^{0}=\frac{1}{2 N_{f}} f_{s}^{0}, \\
f_{d}^{0}=\frac{n_{p}+2 n_{n}}{3\left(n_{p}+n_{n}\right)} f_{v}^{0}+\frac{1}{2 N_{f}} f_{s}^{0}, & f_{\bar{d}}^{0}=\frac{1}{2 N_{f}} f_{s}^{0}, \\
f_{s}^{0}=\frac{1}{2 N_{f}} f_{s}^{0}, & f_{\bar{s}}^{0}=\frac{1}{2 N_{f}} f_{s}^{0},
\end{array}
$$

with $n_{p} / n_{n}=2 / 3$ denoting the proton and neutron fraction of the colliding nuclei. We will vary the parameters $\xi_{0}$ representing the initial momentum anisotropy, as well as $f_{g}^{0}, f_{s}^{0}$, and $f_{v}^{0}$ to investigate the sensitivity of our results to the initial conditions.

Starting from the above initial conditions, we solve the QCD Boltzmann equation [51]

$$
\begin{aligned}
& {\left[\frac{\partial}{\partial \tau}-\frac{p_{\|}}{\tau} \frac{\partial}{\partial p_{\|}}\right] f_{a}\left(\tau, p_{T}, p_{\|}\right)} \\
& \quad=-C_{a}^{2 \leftrightarrow 2}[f]\left(\tau, p_{T}, p_{\|}\right)-C_{a}^{1 \leftrightarrow 2}[f]\left(\tau, p_{T}, p_{\|}\right),
\end{aligned}
$$

for a (longitudinally) boost-invariant (transversely) homogeneous system undergoing a one-dimensional Bjorken expansion. We include all leading-order elastic $\left(C^{2 \leftrightarrow 2}\right)$ and inelastic $\left(C^{1 \leftrightarrow 2}\right)$ interactions, and following previous works $[13,31]$ accounts for in-medium screening of elastic interactions [34] and the Landau-Pomeranchuk-Migdal [52-54] suppression of inelastic rates, as described in detail in Ref. [35].

Nonequilibrium evolution of macroscopic properties. In order to connect the nonequilibrium initial state, with the subsequent hydrodynamic evolution we will focus on the preequilibrium evolution of the macroscopic properties of the QGP. Specifically, we will investigate the evolution of the energy-momentum tensor $T^{\mu \nu}$ and conserved currents $J_{f}^{\mu}$, determined, respectively, as

$$
\begin{aligned}
T^{\mu \nu} & =\int \frac{d^{3} p}{(2 \pi)^{3}} \frac{p^{\mu}}{p} p^{\nu}\left[\nu_{g} f_{g}(\vec{p})+\nu_{q} \sum_{f}\left[f_{q_{f}}(\vec{p})+f_{\bar{q}_{f}}(\vec{p})\right]\right], \\
\Delta J_{f}^{\mu} & =\int \frac{d^{3} p}{(2 \pi)^{3}} \frac{p^{\mu}}{p}\left\{\nu_{q}\left[f_{q_{f}}(\vec{p})-f_{\bar{q}_{f}}(\vec{p})\right]\right\} .
\end{aligned}
$$

Based on the symmetries of the one-dimensional Bjorken expansion, one finds that in Milne $(\tau, x, y, \eta)$ coordinates $T^{\mu \nu}=\operatorname{diag}\left(e, p_{T}, p_{T}, p_{L} / \tau^{2}\right)$ and $\Delta J_{f}^{\nu}=\left(\Delta n_{f}, 0,0,0\right)$ in the local rest frame $u^{\mu}=(1,0,0,0)[55]$ and the conservation laws take the form

$$
\partial_{\tau} e+\frac{e+p_{L}}{\tau}=0, \quad \partial_{\tau} \Delta n_{f}+\frac{\Delta n_{f}}{\tau}=0,
$$

such that, irrespective of the underlying microscopic dynamics, the charge density per unit rapidity $\tau \Delta n_{f}=$ $\left(\tau \Delta n_{f}\right)_{0}=\left(\tau \Delta n_{f}\right)_{\text {eq }}$ remains constant throughout the evolution. Conversely, the evolution of the energy density $e$ is affected by the longitudinal pressure $p_{L}$. Because of the rapid longitudinal expansion, the longitudinal pressure $p_{L} \ll e$ is initially much smaller than the transverse pressure $p_{T} \simeq e / 2$, resulting in a constant ratio of energy per baryon $e / \Delta n_{B}$ and a constant energy density per unit rapidity $(e \tau)_{0}$ at early times.

Over the course of the nonequilibrium evolution, the longitudinal expansion slows down, while kinetic interactions lead to a continuous increase of the longitudinal 

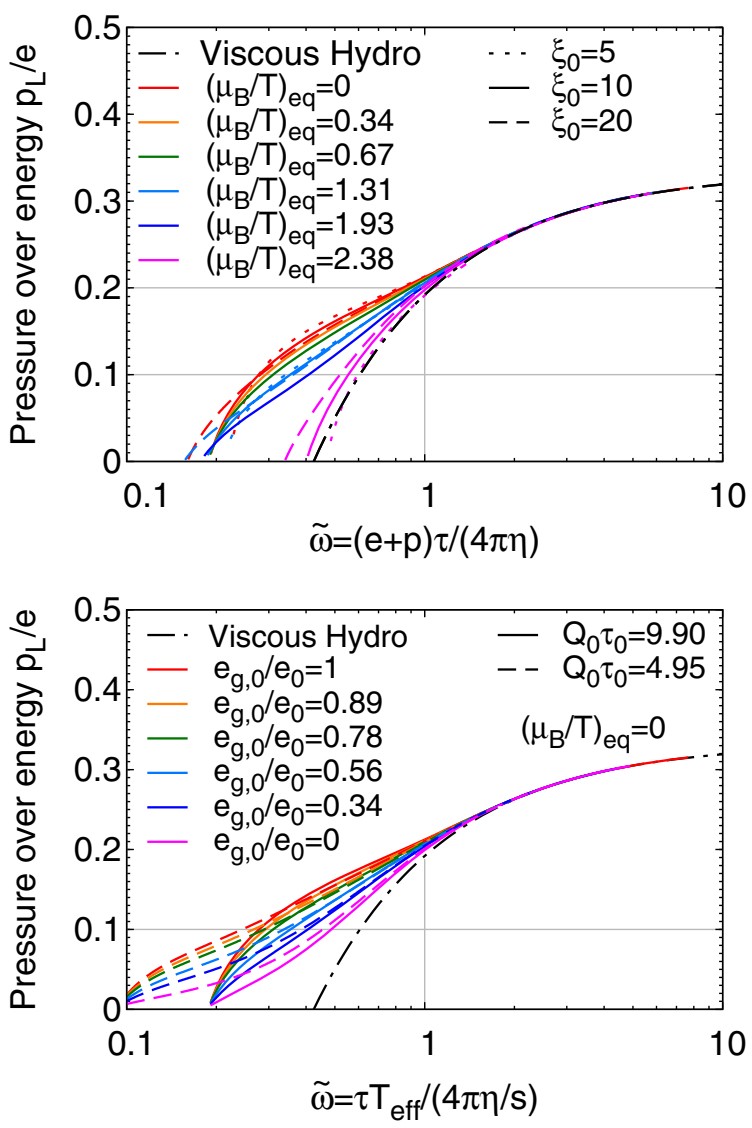

FIG. 1. Nonequilibrium evolution of the longitudinal pressure over energy ratio $p_{L} / e$ for different net-baryon density (top) and different initial quark to gluon ratios at zero density (bottom). Dotted and dashed curves show results for different initial anisotropies $\xi_{0}$ (top) and different initialization times $Q_{s} \tau_{0}$ (bottom).

pressure $p_{L}$ (cf. Fig. 1). Eventually, the residual deviations from equilibrium can be captured by hydrodynamic constitutive relations, where to first order in the gradient expansion the longitudinal pressure is determined by

$$
\frac{p_{L}}{e}=\frac{1}{3}-\frac{16}{9} \frac{\eta}{(e+p) \tau}
$$

with shear viscosity $\eta$ and thermodynamic pressure $p=e / 3$ for a conformal plasma. Ultimately, the QGP approaches an isotropic equilibrium state $\left(p_{L}=p_{T}=e / 3\right)$, where energy and charge densities are described by temperature $T$ and chemical potentials $\mu_{f}$ as

$$
\begin{aligned}
e_{\mathrm{eq}} & =\left[\nu_{g} \frac{\pi^{2}}{30}+\nu_{q} \frac{\pi^{2}}{120} \sum_{f}\left(7+\frac{30}{\pi^{2}} \frac{\mu_{f}^{2}}{T^{2}}+\frac{15}{\pi^{4}} \frac{\mu_{f}^{4}}{T^{4}}\right)\right] T^{4}, \\
\Delta n_{f, e q} & =\frac{\nu_{q}}{6}\left[\frac{\mu_{f}}{T}+\frac{1}{\pi^{2}} \frac{\mu_{f}^{3}}{T^{3}}\right] T^{3}
\end{aligned}
$$

respectively, for an ultrarelativistic plasma of quarks and gluons, such that $\tau^{4 / 3} e$ and $\mu_{f} / T$ approach constant values $\left(\tau^{4 / 3} e\right)_{\mathrm{eq}}$ and $\left(\mu_{f} / T\right)_{\mathrm{eq}}$, respectively, at late times $\tau \gg \eta /(e+p)$ when the QGP approaches local thermal equilibrium.

We present our results for the nonequilibrium evolution of the longitudinal pressure to energy density ratio in Fig. 1, where we show the evolution of $p_{L} / e$ for different initial conditions at zero and nonzero net-baryon density summarized in Table I. Starting from a highly anisotropic initial state at early times, the longitudinal pressure soon exhibits a rapid rise, followed by a slow approach toward isotropy $\left(p_{L} / e=1 / 3\right)$ at late times.

Strikingly, previous studies of the pressure isotropization in pure glue QCD [9,10,31] and QCD at zero density [13] have shown that the nonequilibrium evolution of the energy-momentum tensor at different coupling strengths is governed by a universal scaling variable $\tilde{w}=\tau T / 4 \pi \eta / \mathrm{s}$ $[11,28]$, representing the ratio of the evolution time $\tau$ to the equilibrium relaxation time $\tau_{R}=4 \pi \eta / s / T$. By introducing an effective temperature $T_{\text {eff }}=\left[30 e(\tau) / \pi^{2} \nu_{\text {eff }}\right]^{1 / 4}$ with $\nu_{\text {eff }}=\nu_{g}+(7 / 4) \nu_{q} N_{f}$ characterizing the energy density of the nonequilibrium QGP, the definition of the scaling variable can be generalized to finite density systems as

$$
\tilde{\omega}=\frac{(e+p) \tau}{4 \pi \eta}=\left(\frac{e+p}{\eta T_{\text {eff }}}\right) \frac{\tau T_{\text {eff }}}{4 \pi}
$$

such that the hydrodynamic evolution of the pressure in Eq. (6) remains a universal function of the scaling variable $p_{L} / e=(1 / 3)-(4 / 9 \pi \tilde{\omega})$, irrespective of the net-baryon density. Indeed, one observes from Fig. 1 that eventually all curves converge toward the same viscous hydrodynamic behavior, which provides an accurate description of the pressure anisotropy for $\tilde{w} \gtrsim 1$. We note that, albeit the dimensionless transport coefficient $\left(\eta T_{\text {eff }} / e+p\right)$, which at zero density reduces to $\eta / s$, exhibits a chemical potential dependence, we find that the variations of $\left(\eta T_{\mathrm{eff}} / e+p\right)$ are only at the $10 \%$ level for the considered range of chemical potentials $\left(\mu_{B} / T\right)_{\mathrm{eq}} \lesssim 2.5$, as can be seen from Table I, where we provide the results for $\left(\eta T_{\text {eff }} / e+p\right)$ extracted from fits to the late time asymptotics of $p_{L} / e$ in Eq. (6).

Despite their common late time behavior, important differences emerge in the evolution of the pressure anisotropy at intermediate times $(\tilde{w} \lesssim 1)$. By comparing the solid and dashed curves in the top panel in Fig. 1, we find that variations of the initial momentum anisotropy $\xi_{0}$ do not significantly affect the evolution of the pressure beyond very early times, which is in line with a detailed earlier investigation in pure glue QCD [12]. Conversely, changes in the chemical composition of the primordial plasma do have a noticeable effect on the isotropization of the pressure at intermediate times, resulting in a moderate dependence on the net-baryon density $\mu_{B} / T$ shown in the 
top panel in Fig. 1. Generally, the isotropization of the pressure proceeds more slowly for a larger quark fraction, which is in line with early theoretical expectations [56], attributing the slower equilibration of the quark sector to the differences in color factors in elastic and inelastic scattering processes. Even in zero density systems, shown in the bottom panel in Fig. 1, the initial quark/gluon ratio affects the evolution of the pressure at intermediate times $\tilde{w} \lesssim 1$, challenging the existence of a universal nonequilibrium attractor, where the evolution of the pressure anisotropy becomes insensitive to the details of the initial conditions before the onset of hydrodynamic behavior $[12,18,20,23,31]$. Nevertheless, the variations of $p_{L} / e$ are still rather moderate, and it is equally important to point out that by the time $\tilde{w} \sim 1$, where hydrodynamics becomes applicable, differences in the initial chemical composition no longer affect the pressure evolution.

Connecting the initial state to hydrodynamics.-Now that we have established the evolution of the pressure anisotropy during the early preequilibrium phase, we can determine how the initial energy density $(e \tau)_{0}$ and netcharge density $\left(\Delta n_{f} \tau\right)_{0}$ affect the initial conditions for the subsequent hydrodynamic evolution. By following the arguments of Ref. [28], the evolution of the conserved quantities $e$ and $\Delta n_{f}$ during the preequilibrium phase can be compactly expressed as

$$
\begin{aligned}
& \left(\tau^{4 / 3} e\right)(\tilde{\omega})=\left(4 \pi \frac{\eta T_{\mathrm{eff}}}{e+p}\right)^{4 / 9}\left(\frac{\pi^{2} \nu_{\mathrm{eff}}}{30}\right)^{1 / 9}(e \tau)_{0}^{8 / 9} C_{\infty} \mathcal{E}(\tilde{\omega}), \\
& \left(\tau \Delta n_{f}\right)(\tilde{\omega})=\left(\tau \Delta n_{f}\right)_{0},
\end{aligned}
$$

respectively, where, as explained in Supplemental Material [57], the function $\mathcal{E}(\tilde{\omega})=\tau^{4 / 3} e(\tau) /\left(\tau^{4 / 3} e\right)_{\mathrm{eq}}$ describes the nontrivial evolution of the energy density due to work performed against the longitudinal expansion $[28,58,59]$. Specifically, at early and late times $\mathcal{E}(\tilde{\omega})$ has the asymptotic behavior

$$
\begin{aligned}
& \left.\mathcal{E}(\tilde{\omega} \gg 1) \simeq 1-\frac{2}{3 \pi \tilde{\omega}} \quad \text { (visc.hydro }\right) \\
& \left.\mathcal{E}(\tilde{\omega} \ll 1) \simeq C_{\infty}^{-1} \tilde{\omega}^{4 / 9} \quad \text { (free streaming }\right)
\end{aligned}
$$

such that the constant $C_{\infty}$ in Eqs. (9) and (10) describes the efficiency with which the initial energy density per unit rapidity $(e \tau)_{0}$ is converted into the thermal energy density at the onset of hydrodynamics.

Because of the different evolution of the pressure anisotropy, this conversion is different between zero and finite density systems, as can be seen in Fig. 2, where we present our results for the nonequilibrium evolution of the energy density of the QGP at different values of the baryon chemical potential $\left(\mu_{B} / T\right)_{\mathrm{eq}}$ at late times. Evidently, the more baryon-rich systems experience a smaller longitudinal pressure during the preequilibrium phase and, therefore,

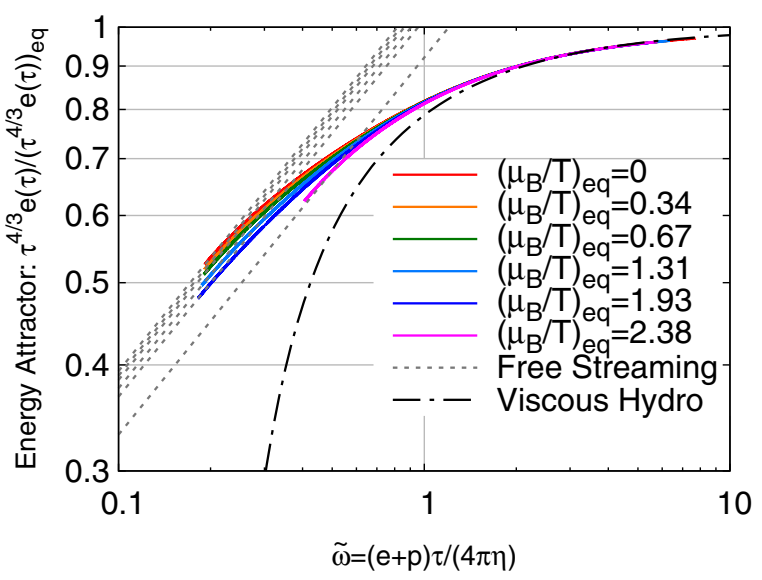

FIG. 2. Nonequilibrium evolution of the energy density for different net-baryon densities, characterized by the ratio of the chemical potentials to temperature $\left(\mu_{B} / T\right)_{\text {eq }}$ at late times. Dashed and solid curves show a comparison to early and late time asymptotics in Eq. (10).

convert the initial energy density more efficiently into thermal energy. By matching the results from QCD kinetic theory simulations to the asymptotic behavior in Eq. (10), we have extracted the values of $C_{\infty}$ to quantify this effect and provide the respective values for all simulations in Table I. We find that, for the considered range of parameters, the dependence on the chemical composition of the primordial plasma is typically on the 10\%-15\% level, indicating that, despite the differences in the pressure evolution, the energy density at the beginning of the hydrodynamics phase can still be estimated to rather good accuracy.

Phenomenological consequences.-We conclude our analysis by studying phenomenological consequences of our results for the dynamical description of heavy-ion collision experiments at RHIC and LHC energies. Neglecting the entropy production during the late-stage hydrodynamic expansion, we follow previous works $[28,60]$ matching the asymptotic entropy density

$(\tau s)_{\mathrm{eq}}=\frac{4}{3} \frac{\left(e \tau^{4 / 3}\right)_{\mathrm{eq}}}{\left(\tau^{1 / 3} T\right)_{\mathrm{eq}}}-\left(\frac{\mu_{B}}{T}\right)_{\mathrm{eq}} \tau \Delta n_{B}-\left(\frac{\mu_{Q}}{T}\right)_{\mathrm{eq}} \tau \Delta n_{Q}$

to the experimentally measured charged particle multiplicities, according to $d N_{\mathrm{ch}} / d \eta=\left(N_{\mathrm{ch}} / S\right)(\tau s)_{\mathrm{eq}} S_{\perp}$, and fix the final entropy to baryon number ratio $(\tau s)_{\mathrm{eq}} /\left(\tau \Delta n_{B}\right)_{\mathrm{eq}}$, according to $s / \Delta n_{B}=144,285$, and 420 for 62.4, 130, and $200 \mathrm{GeV} \mathrm{Au}+\mathrm{Au}$ collisions, respectively. We employ $S / N_{\mathrm{ch}}=8.36[28,61]$ and estimate the transverse area $S_{\perp}=\pi R_{A}^{2}=128,138$, and $95 \mathrm{fm}^{2}$ for central $\mathrm{Au}+\mathrm{Au}$, $\mathrm{Pb}+\mathrm{Pb}$, and $\mathrm{Xe}+\mathrm{Xe}$ collisions, respectively, with $d N_{\mathrm{ch}} / d \eta=470,590$, and 665 for 62.4, 130, and $200 \mathrm{GeV} \mathrm{Au}+\mathrm{Au}$ collisions, respectively; $d N_{\mathrm{ch}} / d \eta=$ 1600 and 1942 for 2.76 and $5.02 \mathrm{TeV} \mathrm{Pb}+\mathrm{Pb}$ collisions, 
respectively; and $d N_{\mathrm{ch}} / d \eta=1166$ for a $5.44 \mathrm{TeV} \mathrm{Xe}+\mathrm{Xe}$ collision [62-64]. Since the overall normalization is fixed by this procedure, Eq. (9) then provides the evolution of the energy $e$ and charge densities $\Delta n_{f}$, from which we infer temperatures $T$ and baryon chemical potential $\mu_{B}$ based on the usual Landau matching procedure associating the conserved charges with their equilibrium values $e=$ $e_{\mathrm{eq}}\left(T, \mu_{f}\right)$ and $\Delta n_{f}=n_{\mathrm{eq}}\left(T, \mu_{f}\right)$.

We illustrate the resulting nonequilibrium trajectories of the QGP in the QCD phase diagram in Fig. 3, where dashed (dotted) lines represent the prehydrodynamic evolution of $\left(T, \mu_{B}\right)$ for $0.2<\tilde{\omega}<1$, whereas solid lines show the hydrodynamic trajectories for $\tilde{\omega}>1$ for two different values of the transport coefficient $\eta T_{\text {eff }} /(e+p)=$ 0.08 (0.16). Strikingly, one observes at first sight that the preequilibrium plasma can exhibit much higher temperatures and chemical potentials as can be achieved during the subsequent hydrodynamic evolution. While in highenergy collisions of heavy nuclei the trajectories run straight down along the vertical axis $\left(\mu_{B} / T \approx 0\right)$, the nonequilibrium trajectories at lower energies bend toward larger values of $\mu_{B} / T$ and deviate significantly from the (perturbative) isentropes indicated by gray dotted lines.

Concerning the applicability of hydrodynamics, one finds that, e.g., in high-energy $\mathrm{Pb}+\mathrm{Pb}$ collisions, hydrodynamics becomes applicable on timescales

$\tau \simeq 1.3 \mathrm{fm} / c\left(\frac{4 \pi \eta / s}{2}\right)^{3 / 2}\left(\frac{d N_{\mathrm{ch}} / d \eta}{1942}\right)^{-1 / 2}\left(\frac{S_{\perp}}{138 \mathrm{fm}^{2}}\right)^{1 / 2}$

and temperatures

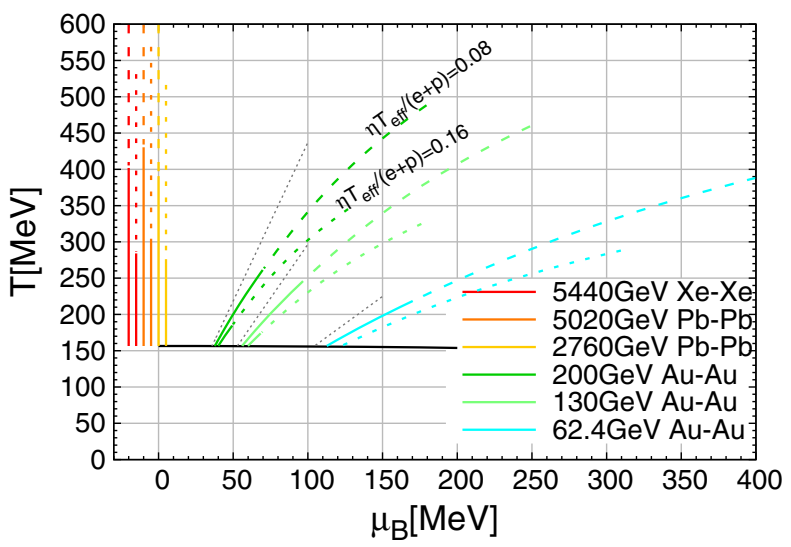

FIG. 3. Nonequilibrium trajectories of temperature $T$ and baryon chemical potential $\mu_{B}$ in the QCD phase diagram for 0\%-5\% most central heavy-ion collisions. Dashed (dotted) lines show the preequilibrium evolution $0.2<\tilde{w}<1$ for $\eta T_{\text {eff }} /(e+p)=0.08(0.16)$; solid lines correspond to the hydrodynamic evolution for $\tilde{w}>1$ which approaches the (perturbative) isentropes indicated by gray dashed lines. The black solid line indicates the QCD crossover temperature $T_{c}=156.5 \mathrm{MeV}$ [65].
$T \simeq 300 \mathrm{MeV}\left(\frac{4 \pi \eta / s}{2}\right)^{-1 / 2}\left(\frac{d N_{\mathrm{ch}} / d \eta}{1942}\right)^{1 / 2}\left(\frac{S_{\perp}}{138 \mathrm{fm}^{2}}\right)^{-1 / 2}$

well above the QCD crossover temperature $T_{c} \simeq 156 \mathrm{MeV}$ [65], indicated by a black line in Fig. 3. However, at lower energies, the nonequilibrium trajectories extend almost all the way to the QCD phase boundary, indicating that the QGP created in low-energy collisions may be significantly out of equilibrium for a substantial part of its lifetime. Even though the use of a perturbative description and the modeling of the space-time dynamics in terms of a onedimensional Bjorken expansion become increasingly questionable at lower energies, we still believe that Fig. 3 points to one of several important challenges in describing the space-time evolution of heavy-ion collisions at low energies [3,66-69].

Conclusions and outlook.-We investigated the early time preequilibrium dynamics of the QGP at zero and finite densities of the conserved baryon and electric charge based on an effective kinetic description of high-energy QCD. We find that, for a plasma undergoing a one-dimensional Bjorken expansion, the nonequilibrium evolution of the energy-momentum tensor can be well described by viscous hydrodynamics for time $\tau \gtrsim 4 \pi \eta / e+p$, which is in accordance with earlier findings at zero density [13]. However, the chemical composition of the QGP can have a significant impact on the evolution of the pressure at early times $\tau \lesssim 4 \pi \eta / e+p$, which is in sharp contrast to the results obtained for single-component plasmas [7-12, $17,18,22,23,25-27,70,71]$, where macroscopic properties such as $p_{L} / e$ exhibit a rapid memory loss at early times, resulting in a universal approach toward viscous hydrodynamics. Even though the ensuing differences in the pressure affect the late time evolution of the QGP only at the $10 \%-15 \%$ level, it is clear that further progress in the theoretical understanding hinges to a considerable extent on an improved determination of the chemical composition of the primordial plasma, created immediately after the collision of heavy nuclei.

We further discussed how the results presented in this Letter can be used to connect the properties of the nonequilibrium initial state to the initial conditions for the subsequent hydrodynamic evolution. By extending previous works [28] to include the effects of finite net-baryon and electric charge density, our calculations thus provide a first important step toward including the evolution of all QCD conserved charges into dynamical descriptions of the preequilibrium stage of heavy-ion collisions [10,31,72]. Beyond such applications to bulk phenomenology, our QCD kinetic theory studies also provide the basis for future investigations of heavy flavor dynamics or the emission of electromagnetic probes, which could provide sensitive probes of the early time nonequilibrium dynamics [73-75]. 
We thank Giuliano Giacalone and Aleksas Mazeliauskas for many insightful discussions and valuable contributions to the Supplemental Material [57]. We further thank Aleksi Kurkela, Jean-Francois Paquet, Ismail Soudi, and Derek Teaney for insightful discussions and collaboration on related projects. This work is supported by the Deutsche Forschungsgemeinschaft (DFG, German Research Foundation) Project No. 315477589 TRR 211. The authors also acknowledge computing time provided by the Paderborn Center for Parallel Computing (PC2) and the National Energy Research Scientific Computing Center, a Department of Energy Office of Science User Facility supported by the Office of Science of the U.S. Department of Energy under Contract No. DE-AC02-05CH11231.

[1] S. Schlichting and D. Teaney, Annu. Rev. Nucl. Part. Sci. 69, 447 (2019).

[2] J. Berges, M. P. Heller, A. Mazeliauskas, and R. Venugopalan, arXiv:2005.12299.

[3] C. Gale, S. Jeon, and B. Schenke, Int. J. Mod. Phys. A 28, 1340011 (2013).

[4] U. Heinz and R. Snellings, Annu. Rev. Nucl. Part. Sci. 63, 123 (2013).

[5] P. M. Chesler and L. G. Yaffe, Phys. Rev. Lett. 102, 211601 (2009).

[6] V. Balasubramanian, A. Bernamonti, J. de Boer, N. Copland, B. Craps, E. Keski-Vakkuri, B. Muller, A. Schafer, M. Shigemori, and W. Staessens, Phys. Rev. Lett. 106, 191601 (2011).

[7] M. P. Heller, R. A. Janik, and P. Witaszczyk, Phys. Rev. Lett. 108, 201602 (2012).

[8] L. Keegan, A. Kurkela, P. Romatschke, W. van der Schee, and Y. Zhu, J. High Energy Phys. 04 (2016) 031.

[9] A. Kurkela and E. Lu, Phys. Rev. Lett. 113, 182301 (2014).

[10] A. Kurkela, A. Mazeliauskas, J.-F. Paquet, S. Schlichting, and D. Teaney, Phys. Rev. Lett. 122, 122302 (2019).

[11] M. P. Heller, A. Kurkela, M. Spaliński, and V. Svensson, Phys. Rev. D 97, 091503(R) (2018).

[12] D. Almaalol, A. Kurkela, and M. Strickland, Phys. Rev. Lett. 125, 122302 (2020).

[13] A. Kurkela and A. Mazeliauskas, Phys. Rev. D 99, 054018 (2019).

[14] J.-P. Blaizot and L. Yan, arXiv:2006.08815.

[15] J.-P. Blaizot and L. Yan, Ann. Phys. (Amsterdam) 412, 167993 (2020).

[16] J.-P. Blaizot and L. Yan, Phys. Lett. B 780, 283 (2018).

[17] S. Kamata, M. Martinez, P. Plaschke, S. Ochsenfeld, and S. Schlichting, Phys. Rev. D 102, 056003 (2020).

[18] M. Strickland, J. High Energy Phys. 12 (2018) 128.

[19] M. Martinez, R. Ryblewski, and M. Strickland, Phys. Rev. C 85, 064913 (2012).

[20] A. Kurkela, W. van der Schee, U. A. Wiedemann, and B. Wu, Phys. Rev. Lett. 124, 102301 (2020).

[21] A. Behtash, S. Kamata, M. Martinez, T. Schäfer, and V. Skokov, Phys. Rev. D 103, 056010 (2021).

[22] M. P. Heller and M. Spalinski, Phys. Rev. Lett. 115, 072501 (2015).

[23] P. Romatschke, Phys. Rev. Lett. 120, 012301 (2018).
[24] A. Behtash, S. Kamata, M. Martinez, and H. Shi, Phys. Rev. D 99, 116012 (2019).

[25] M. P. Heller, R. Jefferson, M. Spaliński, and V. Svensson, Phys. Rev. Lett. 125, 132301 (2020).

[26] G. S. Denicol and J. Noronha, Phys. Rev. Lett. 124, 152301 (2020).

[27] M. Strickland, J. Noronha, and G. S. Denicol, Phys. Rev. D 97, 036020 (2018).

[28] G. Giacalone, A. Mazeliauskas, and S. Schlichting, Phys. Rev. Lett. 123, 262301 (2019).

[29] P. Romatschke, J. High Energy Phys. 12 (2017) 079.

[30] G. S. Denicol and J. Noronha, Phys. Rev. D 97, 056021 (2018).

[31] A. Kurkela, A. Mazeliauskas, J.-F. Paquet, S. Schlichting, and D. Teaney, Phys. Rev. C 99, 034910 (2019).

[32] T. N. da Silva, D. Chinellato, M. Hippert, W. Serenone, J. Takahashi, G. S. Denicol, M. Luzum, and J. Noronha, Phys. Rev. C 103, 054906 (2021).

[33] C. Gale, J.-F. Paquet, B. Schenke, and C. Shen, Nucl. Phys. A1005, 121863 (2021).

[34] P. B. Arnold, G. D. Moore, and L. G. Yaffe, J. High Energy Phys. 01 (2003) 030.

[35] X. Du and S. Schlichting, companion paper, Phys. Rev. D 104, 054011 (2021).

[36] F. Gelis, E. Iancu, J. Jalilian-Marian, and R. Venugopalan, Annu. Rev. Nucl. Part. Sci. 60, 463 (2010).

[37] E. Iancu and R. Venugopalan, in Quark-Gluon Plasma 3, edited by R. C. Hwa and X.-N. Wang (World Scientific, Singapore, 2003), pp. 249-363, https://doi.org/10.1142/ 9789812795533_0005.

[38] A. Kovner, L. D. McLerran, and H. Weigert, Phys. Rev. D 52, 6231 (1995).

[39] A. Krasnitz and R. Venugopalan, Nucl. Phys. B557, 237 (1999).

[40] A. Krasnitz, Y. Nara, and R. Venugopalan, Nucl. Phys. A727, 427 (2003).

[41] J.-P. Blaizot, T. Lappi, and Y. Mehtar-Tani, Nucl. Phys. A846, 63 (2010).

[42] B. Schenke, P. Tribedy, and R. Venugopalan, Phys. Rev. C 86, 034908 (2012).

[43] F. Gelis and N. Tanji, J. High Energy Phys. 02 (2016) 126.

[44] H. Fujii, F. Gelis, and R. Venugopalan, Nucl. Phys. A780, 146 (2006).

[45] F. Gelis, K. Kajantie, and T. Lappi, Phys. Rev. Lett. 96, 032304 (2006).

[46] L. D. McLerran, S. Schlichting, and S. Sen, Phys. Rev. D 99, 074009 (2019).

[47] M. Lushozi, L. D. McLerran, M. Praszalowicz, and G. Yu, Phys. Rev. C 102, 034908 (2020).

[48] K. Kajantie, L. D. McLerran, and R. Paatelainen, Phys. Rev. D 101, 054012 (2020).

[49] P. Agostini, T. Altinoluk, and N. Armesto, Eur. Phys. J. C 79, 600 (2019).

[50] We describe the preequilibrium evolution in terms of proper time $\tau=\sqrt{t^{2}-z^{2}}$ and rapidity $\eta=\operatorname{atanh}(z / t)$ coordinates, along with the transverse $p_{T}=\sqrt{p_{x}^{2}+p_{y}^{2}}$ and longitudinal momenta $p_{\|} \equiv p^{\eta} / \tau=p_{T} \sinh \left(y_{p}-\eta\right)$ momenta in comoving coordinates $\left[y_{p}=\operatorname{atanh}\left(p_{z} / p\right)\right]$. Since the preequilibrium stage lasts only for $\sim 1 \mathrm{fm} / c$, we follow the 
concept of Refs. [10,31] in neglecting the transverse expansion which becomes important at later times and treating the preequilibrium QGP as locally homogenous in the transverse plane.

[51] A. H. Mueller, Phys. Lett. B 475, 220 (2000).

[52] L. Landau and I. Pomeranchuk, Dokl. Akad. Nauk Ser. Fiz. 92, 735 (1953).

[53] L. Landau and I. Pomeranchuk, Dokl. Akad. Nauk Ser. Fiz. 92, 535 (1953).

[54] A. B. Migdal, Dokl. Akad. Nauk Ser. Fiz. 105, 77 (1955).

[55] Note that, due to the symmetries of Bjorken flow, there is no ambiguity in the choice of the local rest frame due to multiple conserved quantities.

[56] T. Biro and J. Zimanyi, Phys. Lett. B 113, 6 (1982).

[57] See Supplemental Material at http://link.aps.org/ supplemental/10.1103/PhysRevLett.127.122301 for derivation of $e$ attractor solution.

[58] J. D. Bjorken, Phys. Rev. D 27, 140 (1983).

[59] M. Gyulassy and T. Matsui, Phys. Rev. D 29, 419 (1984).

[60] J. Jankowski, S. Kamata, M. Martinez, and M. Spaliński, arXiv:2012.02184.

[61] P. Hanus, K. Reygers, and A. Mazeliauskas, Phys. Rev. C 100, 064903 (2019).

[62] I. Arsene et al. (BRAHMS Collaboration), Nucl. Phys. A757, 1 (2005).
[63] B. Back et al. (PHOBOS Collaboration), Phys. Rev. C 74, 021901 (2006).

[64] K. Aamodt et al. (ALICE Collaboration), Phys. Rev. Lett. 106, 032301 (2011).

[65] A. Bazavov et al. (HotQCD Collaboration), Phys. Lett. B 795, 15 (2019).

[66] P. Romatschke, Int. J. Mod. Phys. E 19, 1 (2010).

[67] L. Yan, Chin. Phys. C 42, 042001 (2018).

[68] C. Shen and L. Yan, Nucl. Sci. Technol. 31, 122 (2020).

[69] T. Dore, J. Noronha-Hostler, and E. McLaughlin, Phys. Rev. D 102, 074017 (2020).

[70] J. Berges, K. Boguslavski, S. Schlichting, and R. Venugopalan, Phys. Rev. D 89, 074011 (2014).

[71] J. Berges, K. Boguslavski, S. Schlichting, and R. Venugopalan, Phys. Rev. D 89, 114007 (2014).

[72] M. Martinez, M. D. Sievert, D. E. Wertepny, and J. Noronha-Hostler, arXiv:1911.10272.

[73] J. Churchill, L. Yan, S. Jeon, and C. Gale, Phys. Rev. C 103, 024904 (2021).

[74] B. S. Kasmaei and M. Strickland, Phys. Rev. D 99, 034015 (2019).

[75] B. S. Kasmaei and M. Strickland, Phys. Rev. D 102, 014037 (2020). 